\title{
Fauna parasitária de peixes oriundos de "pesque-pague" do município de Franca, São Paulo, Brasil. I. Protozoários
}

\author{
Marcos Tavares-Dias ${ }^{1}$ \\ Maurício L. Martins ${ }^{1}$ \\ Flávio R. Moraes ${ }^{1,2}$
}

\begin{abstract}
Parasitic fauna of cultivated fishes in feefishing farm of Franca, São Paulo State, Brazil. I. Protozoans. The present work studied protozoan parasites of cultivated fishes $(\mathrm{N}=433)$ from two feefishing farm situated in Franca, São Paulo, Brazil, during a period of April 1997 through March 1999. Specimens of piauçú Leporinus macrocephalus Garavello \& Britski, 1988 (Anostomidae), pacu Piaractus mesopotamicus Holmberg, 1887 (Characidae) carp Cyprinus carpio Linnaeus, 1758 (Cyprinidae), Tillapia rendalli Boulenger, 1896 (Cichlidae), nile-tilapia Oreochromis niloticus Linnaeus, 1758 (Cichlidae), matrinxã Brycon cephalus Günther, 1869 (Characidae) and tambacu hybrid (male of $P$. mesopotamicus $\mathrm{x}$ female of Colossoma macropomum Cuvier, 1818) were collected. The fishes were parasitized with protozoans Ichthyophthirius multifiliis Fouquet, 1876 (Protozoa), Trichodina sp. and Piscinoodinium pillulare (Schäperclaus, 1954), Lom, 1981 (Protozoa). In the cold season (autumn and/or winter) all species of fish were infected with I. multifiliis. Higher susceptibility to Trichodina sp. was observed in L. macrocephalus, C. carpio and $P$. mesopotanicus compared to tambacu, $B$. cephalus, $T$. rendalli and $O$. niloticus. It was not observed significant difference $(\mathrm{P}>0.05)$ in the seasonal variation of Trichodina sp. and $P$. pillulare infection of all species. A great number of $P$. pillulare without significant difference $(\mathrm{P}>0.05)$ was reported to $L$. macrocephalus, $P$. mesopotamicus and tambacu.

KEY WORDS. Ichthyophthirius nultifiliis, freshwater fish, parasites, Piscinoodinium pillulare, Trichodina sp.
\end{abstract}

O sistema de cultivo intensivo, usualmente praticado pelas pisciculturas industriais, caracteriza-se pelo aumento da densidade de estocagem de peixes, visando a máxima produção. Assim, nesse tipo de exploração comercial, surgem com freqüência problemas relativos ao manejo inadequado, às questões nutricionais e às enfermidades infecciosas e parasitárias (THATCHER \& BRITES-NETO 1994; MARTINS \& ROMERO 1996; MARTINS 1998).

As parasitoses são as maiores causas de perdas nas pisciculturas industrial ou esportiva, sendo de maior relevância no neotrópico, pelas características climáticas pertinentes à região, que propiciam suas rápida e constante propagação (THATCHER \& BRITES-NETO 1994).

1) Laboratório de Patologia Organismos Aquáticos, Centro de Aqüicultura, Universidade Estadual Paulista. Via de Acesso Prof. Paulo Donato Castellane, 14870-000 Jaboticabal, São Paulo, Brasil. E-mail: mtavares@caunesp.unesp.br

2) Departamento de Patologia Veterinária, FCAV, Universidade Estadual Paulista. Via de Acesso Prof. Paulo Donato Castellane, 14870-000 Jaboticabal, São Paulo, Brasil. 
As enfermidades causadas por protozoários manifestam-se freqüentemente após operações de manejo em épocas frias do ano e outras situações estressantes (CECCARElli et al. 1990; THATCHER \& BRITES-NETO 1994; MARTINS \& ROMERO 1996). Entretanto, baixas temperaturas durante o inverno, em regiões de clima temperado podem, retardar o desenvolvimento de epizootias (MEYER 1970).

Em hábitat em que há constância na temperatura da água, como nas regiões tropicais, as variações sazonais do parasitismo como decorrência de variações de temperatura não são significativas (BAUER \& KARIMOV 1990). Todavia, tilápiasdo-Nilo em cultivo extensivo, em regiões de clima tropical, submetidas a ambiente aquático de má qualidade, estão sujeitas a epizootias pelo protozoário Ichthyophthirius multifiliis Fouquet, 1876 associadas ou não à saprolegniose, resultando em severo quadro de mortalidade (TAVARES-DIAS et al. no prelo), devido ao comprometimento dos mecanismos de defesa do hospedeiro (BAUER \& KARIMOV 1990).

O desenvolvimento de técnicas para evitar transtornos à saúde dos peixes cultivados é objeto de interesse comum entre pesquisadores e criadores. Todavia, o desenvolvimento de tais técnicas passa pela necessidade do diagnóstico da situação epidemiológica e sanitária dos estabelecimentos de cultivo para que se possa interferir de forma eficiente no processo. Em função do exposto, este trabalho visou descrever a prevalência, carga parasitária e a sazonalidade de protozoários, eventuais surtos epizoóticos por tais agentes em peixes cultivados em dois "pesque-pagues" do município de Franca, Estado de São Paulo, durante o período de 24 meses.

\section{MATERIAL E MÉTODOS}

\section{Peixes e Características de Criação}

Mensalmente, durante o período de abril de 1997 a março de 1999, foram colhidos vinte peixes em cada um dos dois "pesque-pagues" amostrados no município de Franca, nordeste do Estado de São Paulo. No pesque-pague 1MG (20 $\left.35^{\prime} 27^{\prime \prime}-47^{\circ} 26^{\prime} 33^{\prime \prime}\right)$ foram necropsiados 226 espécimes e no pesque-pague $2 \mathrm{RB}$ $\left(20^{\circ} 34^{\prime} 50^{\prime \prime}-47^{\circ} 21^{\prime} 29^{\prime \prime}\right) 207$ espécimes. Os peixes foram cultivados intensivamente e alimentados com ração comercial. Todos foram capturados com auxílio de caniço e anzol e submetidos aos exames necroscópico e parasitológico.

\section{Exames necroscópico e parasitológico}

Após a biometria, os peixes foram sacrificados por comoção cerebral e necropsiados, para investigação da eventual presença de alterações estruturais e de parasitos. O exame necroscópico foi realizado de acordo com método de rotina ao mesmo tempo em que se fazia pesquisa parasitológica à fresco. Para tanto foram colhidos o muco da superfície corporal e fragmentos de brânquias. Essas peças, foram banhadas em uma gota de solução salina a $0,65 \%$, comprimidas entre lâmina e lamínula e a preparação examinada sob microscopia de luz. O intestino de cada espécime também foi examinado, com auxílio de estereomicroscópio. A identificação dos parasitos seguiu as recomendações de THATCHER (1991) e MARTINS (1998). A prevalência foi calculada como sendo o número de hospedeiros infectados/número de hospedeiros examinados, de acordo com BusH et al. (1997). A carga parasitária foi definida como o número total de parasitos, de cada espécie, dividido pelo número de hospedeiros. 


\section{Colheita e quantificação dos parasitos}

$\mathrm{O}$ processamento do material foi feito no local ou no Laboratório de Patologia de Organismos Aquáticos, Centro de Aqüicultura da Universidade Estadual Paulista (CAUNESP), Jaboticabal, de acordo com cada tipo de parasito encontrado, após ser devidamente etiquetado e catalogado em ficha de necrópsia. A metodologia empregada para colheita e fixação foi a recomendada por MARTINS \& ROMERO (1996) e EIRAs et al. (2000).

Os protozoários foram quantificados em câmara de Macmaster e microscópia de luz. Após homogenização do material, $0,30 \mathrm{~mL}$ da solução foram colocados na câmara de contagem e em seguida feita a estimativa do número de parasitos.

\section{Tratamentos}

Em ambos os "pesque-pagues", quando constatada elevada infecção parasitária, os peixes foram submetidos a tratamentos de acordo com cada tipo de parasito.

\section{Análise da água dos viveiros e preciptação pluviométrica}

No momento de cada colheita foram avaliados a temperatura da água com termômetro de bulbo; o pH, utilizando-se "peagâmetro" eletrônico "Corning"; condutividade elétrica em condutivímetro "Corning" e a concentração de oxigênio dissolvido em oxímetro "YSY-Mod.50".

Os dados de preciptação pluviométrica foram fornecidos pela Coordenadoria de Assistência Técnica (CAT) da Casa da Agricultura de Franca.

\section{Análise estatística}

Para estudo do efeito da sazonalidade na ocorrência de parasitos utilizou-se a análise de variância, sem repetição. As médias foram comparadas pelo teste de Tuckey, a 5\% de probabilidade (STEEL \& TORRIE 1980).

\section{RESULTADOS}

\section{Análise da água dos viveiros}

Os valores médios da temperatura da água, $\mathrm{pH}$, condutividade elétrica e oxigênio dissolvido, durante o período de abril de 1997 a março de 1999, no "pesque-pague" $1 \mathrm{MG}$ e $2 \mathrm{RB}$ estão demonstrados na figura $1 \mathrm{~A}-\mathrm{B}$. Observa-se por essa figura maior variação nos valores médios da condutividade elétrica da água no "pesque-pague" 2RB, não havendo diferença significativa entre os demais parâmetros estudados.

\section{Prevalência de parasitos e carga parasitária média}

Em ambos "pesque-pagues" foram necropsiados 433 espécimes de teleósteos, no período de abril de 1997 a março de 1999. Desses, 111 eram piauçús Leporinus macrocephalus Garavello \& Britski, 1988 (medindo de 21,5 a 46,0 cm e pesando de 110,0 a 1360 g), 92 pacus Piaractus mesopotamicus Holmberg, 1887 (medindo de 15,0 a 47,0 cm e pesando de 80,0 a 2000,0 g), 9 carpas Cyprinus carpio Linnaeus, 1758 (medindo de 21,0 a 45,5 cm e pesando de 110,0 a 1500,0 g), 9 Tillapia rendalli Boulenger, 1896 (medindo de 16,0 a 23,5 cm e pesando de 80,0 a 250,0 g), 110 tilápias-do-Nilo Oreochromis niloticus Linnaeus, 1757 (medindo de 


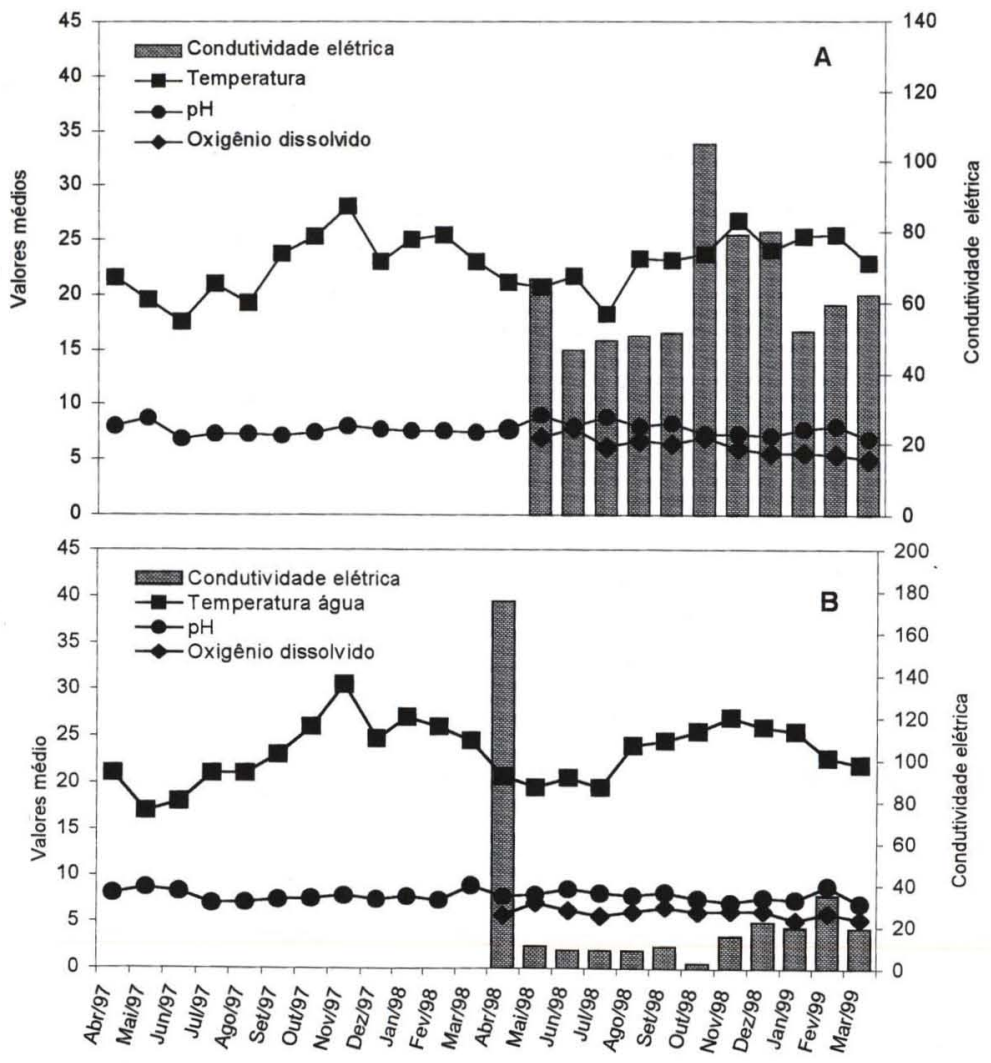

Fig. 1. Valores de condutividade elétrica $(\mu \mathrm{S} / \mathrm{cm})$, temperatura $\left({ }^{\circ} \mathrm{C}\right), \mathrm{pH}$ e teor de oxigênio dissolvido ( $\mathrm{mg} / \mathrm{L}$ ) na água dos viveiros de cultivo no "pesque-pague" $1 \mathrm{MG}(\mathrm{A})$ e "pesque-pague" 2RB (B), tomados em avaliações mensais, durante o período de abril de 1997 a março de 1999, no município de Franca, São Paulo.

20,0 a $34,5 \mathrm{~cm}$ e pesando de 160,0 a $750,0 \mathrm{~g}$ ), 26 matrinxãs Brycon cephalus Günther, 1869 (medindo de 33,5 a 48,0 cm e pesando de 610, 0 a 1980,0 g) e 76 tambacus (macho de $P$. mesopotamicus x fêmea de Colossoma macropomum Cuvier, 1818 (medindo de 17,0 a 50,0 cm e pesando de 100,0 a 2270,0 g).

A carga parasitária média e a prevalência de protozoários nas brânquias de peixes necropsiados $(n=226)$ no "pesque-pague" $1 \mathrm{MG}$ estão expressos na tabela I. Sua análise permite observar que o Ichthyophthirius multifiliis Fouquet, 1876 (Protozoa) infectou somente brânquias de P. mesopotamicus e B. cephalus, e que Trichodina sp. ocorreu em P. mesopotamicus, L. macrocephalus, tambacu e $O$. niloticus. A maior prevalência de Trichodina sp. foi em P. mesopotamicus e L. macrocephalus, mas o primeiro hospedeiro apresentou maior número deste ciliado em seu organismo. Piscinoodinium pillulare (Schäperclaus, 1954) Lom, 1981 (Protozoa) ocorreu somente nas brânquias de P. mesopotamicus, L. macrocephalus e tambacu. A maior prevalência da infecção foi, porém, observada em L. macrocephalus. 
No "pesque-pague" 2RB, foram examinados 207 peixes e a infecção por I. multifiliis ocorreu em tambacu, O. niloticus, L. macrocephalus e em C. carpio, sendo a maior carga parasitária média observada no piauçú. O L. macrocephalus foi a espécie que apresentou maior prevalência de infecção por Trichodina sp., mas o $P$. mesopotamicus albergou maior carga parasitária. As maiores ocorrência e carga de $P$. pillulare foram observadas nas brânquias de $L$. macrocephalus, seguida em $P$. mesopotamicus e tambacu e muito superiores aos encontrados em T. rendalli (Tab. II).

Em 433 peixes necropsiados nos dois "pesque-pagues" de Franca, a infecção por I. multifiliis variou de $2,7 \%$ a $11,1 \%$, ocorrendo em todas as espécies estudadas. A maior carga parasitária ocorreu em L. macrocephalus e a menor em O. niloticus (Tab. III).

Leporinus macrocephalus, P. mesopotamicus e C. carpio foram as espécies mais susceptíveis à infecção por Trichodina sp. quando comparadas ao tambacu, $B$. cephalus, $T$. rendalli e a tilápia-do-Nilo, sendo o maior número desses parasitos observado em pacus (P. mesopotamcus) (Tab. III).

A maior prevalência do protozoário $P$. pillulare foi obervada em $L$. macrocephalus (42,0\%), seguida pelo P. mesopotamicus $(24,0 \%)$ e tambacus $(20,0 \%)$. A carga parasitária por sua vez foi similar entre tais hospedeiros. Esse dinoflagelado teve baixa prevalência e carga parasitária média em $B$. cephalus, estando ausente nas tilápias e carpas (Tab. III).

\section{Variação sazonal dos parasitos}

Infecção por I. multifiliis em L. macrocephalus, P. mesopotamicus e tambacu, ocorreu no outono e inverno, e em O. niloticus, somente nos meses de outono (Fig. 2A). Houve diferença estatisticamente significativa $(\mathrm{P}<0,05)$ entre outono/inverno e primavera/verão. A maior carga média desse parasito em L. macrocephalus ocorreu no outono (Fig. 2B).

Em $P$. mesopotamicus, tambacu e L. macrocephalus a infecção por $P$. pillulare não mostrou diferença estatisticamente significativa $(\mathrm{P}>0,05)$ entre os meses de outono, inverno, primavera e verão. Porém, a carga média de $P$. pillulare em tambacu foi menor no inverno, e L. macrocephalus no outono e inverno, aumentando na primavera (Fig. 3A-B). O menor número de parasitos em $P$. mesopotamicus foi observado no outono $(2316,7)$, aumentando no inverno $(85729,0)$, voltando a decrescer na primavera $(12340,0)$ e chegou ao máximo no verão $(8339835,3)$.

O parasitismo por Trichodina sp., em L. macrocephalus e P. mesopotamicus, ocorreu em todas as estações do ano. Em tambacu, a infecção ocorreu somente nos meses de outono e em O. niloticus, no inverno (Fig. 4A). Entretanto, nessas quatro espécies, não houve diferença estatisticamente significativa $(P>0,05)$ entre as estações do ano. Porém, em L. macrocephalus e em P. mesopotamicus o número médio de Trichodina sp. declinou no inverno e, no P. mesopotamicus, voltou a crescer na primavera (Fig. 4B). 
Tabela I. Carga parasitária média e prevalência (\%) de protozoários em brânquias de 226 peixes necropsiados no "pesque-pague" 1MG, periodo de abril de 1997 a março de 1999, no município de Franca, São Paulo.

\begin{tabular}{|c|c|c|c|c|c|c|c|c|c|c|}
\hline \multirow{2}{*}{$\begin{array}{c}\text { Hospedeiros } \\
\text { Parasitos }\end{array}$} & \multicolumn{2}{|c|}{ P. mesopotamicus $(\mathrm{N}=44)$} & \multicolumn{2}{|c|}{ L. macrocephalus $(\mathrm{N}=29)$} & \multicolumn{2}{|c|}{ Tambacu $(\mathrm{N}=20)$} & \multicolumn{2}{|c|}{ B. cephalus $(\mathrm{N}=5)$} & \multicolumn{2}{|c|}{ O. niloticus $(\mathrm{N}=7$ ) } \\
\hline & Carga parasitária & $\%$ & Carga parasitária & $\%$ & Carga parasitária & $\%$ & Carga parasitária & $\%$ & Carga parasitária & $\%$ \\
\hline 1. multifiliis & $8315,4 \pm 1287,0$ & 11,0 & - & - & - & - & $222,0 \pm 11,0$ & 9,0 & - & - \\
\hline Trichodina sp. & $1427,1 \pm 254,4$ & 13,0 & $310 \pm$ & 12,0 & $248,0 \pm 12,9$ & 2,0 & - & - & $243,0 \pm 106,9$ & 8,0 \\
\hline$P$. pillulare & $16494,0 \pm 1517,0$ & 6,0 & $1306673,0 \pm 41144,0$ & 17,2 & $2283,0 \pm 364,6$ & 4,3 & $879,0 \pm 39,9$ & 14,3 & - & - \\
\hline
\end{tabular}

Tabela II. Carga parasitária média e prevalência (\%) de protozoários em braânquias de 207 peixes necropsiados no "pesque-pague" 2RB, período de abril de 1997 a março de 1999, no município de Franca, São Paulo.

\begin{tabular}{|c|c|c|c|c|c|c|c|c|c|c|c|c|}
\hline \multirow{2}{*}{$\begin{array}{c}\text { Hospedeiros } \\
\text { Parasitos }\end{array}$} & \multicolumn{2}{|c|}{ P. mesopotamicus $(\mathrm{N}=29)$} & \multicolumn{2}{|c|}{ L. macrocephalus $(\mathrm{N}=49)$} & \multicolumn{2}{|l|}{ Tambacu $(\mathrm{N}=17)$} & \multicolumn{2}{|c|}{ T. rendalli $(\mathrm{N}=1)$} & \multicolumn{2}{|c|}{ O. niloticus $(\mathrm{N}=3)$} & \multicolumn{2}{|c|}{ C. carpio $(n=4)$} \\
\hline & Carga parasitária & $\%$ & Carga parasitária & $\%$ & Carga parasitária & $\%$ & Carga parasitária & $\%$ & Carga parasitária & $\%$ & Carga parasitária & $\%$ \\
\hline 1. multifiliis & - & - & $442100,3 \pm$ & 11,0 & $11747,7 \pm$ & 8,0 & - & - & $76,0 \pm 44,9$ & 4,0 & $81778,0 \pm 98,0$ & 12,0 \\
\hline Trichodina sp. & $313705,0 \pm 61197,0$ & 13,0 & $766,1 \pm$ & 22,0 & - & & - & - & - & - & $128,0 \pm 12,0$ & 12,0 \\
\hline P. pillulare & $5568396,0 \pm 1639261,2$ & 60,0 & $7087989,9 \pm 2211363,0$ & 70,0 & $7545925,0 \pm 1479255,4$ & 38,0 & - & - & - & - & - & - \\
\hline
\end{tabular}

Tabela III. Carga parasitária média e prevalência (\%) de protozoários em brânquias de 433 peixes necropsiados no periodo de abril de 1997 a março de 1999, em "pesque-pagues" do municipio de Franca, São Paulo.

\begin{tabular}{|c|c|c|c|c|c|c|c|c|c|c|c|c|c|c|c|c|c|c|}
\hline \multirow{2}{*}{$\begin{array}{c}\text { Hospedeiros } \\
\text { Parasitos }\end{array}$} & \multicolumn{3}{|c|}{$\begin{array}{l}\text { P. mesopotamicus } \\
(\mathrm{N}=73)\end{array}$} & \multicolumn{3}{|c|}{$\begin{array}{l}\text { L. macrocephalus } \\
\qquad(\mathrm{N}=78)\end{array}$} & \multicolumn{3}{|c|}{$\begin{array}{l}\text { Tambacu } \\
(\mathrm{N}=37)\end{array}$} & \multicolumn{3}{|c|}{$\begin{array}{l}\text { B. cephalus } \\
(\mathrm{N}=7)\end{array}$} & \multicolumn{2}{|l|}{$\begin{array}{l}\text { T. rendalli } \\
(\mathrm{N}=2)\end{array}$} & \multicolumn{2}{|l|}{$\begin{array}{l}\text { O. niloticus } \\
(\mathrm{N}=10)\end{array}$} & \multicolumn{2}{|l|}{$\begin{array}{l}\text { C. carpio } \\
(n=4)\end{array}$} \\
\hline & Carga paras & sitária & $\%$ & Carga paras & sitária & $\%$ & Carga para & asitária & $\%$ & Carga para & asitáric & $a \%$ & Carga parasitária & $\%$ & Carga parasitária & $\%$ & Carga parasitária & $\%$ \\
\hline 1. multifiliis & $8315,4 \pm$ & 1287,0 & 9,0 & $442100,3 \pm$ & 85646,5 & 6,0 & $11747,7 \pm$ & 1984,9 & 3,5 & $222,0 \pm$ & 10,0 & 6,2 & $879,0 \pm 22,0$ & 11,1 & $76,0 \pm 44,9$ & 2,7 & $1778,0 \pm 1230,0$ & 11,1 \\
\hline Trichodina sp. 1 & $105519,8 \pm$ & 35937,9 & 13,0 & $248,0 \pm$ & 145,4 & 17,0 & $248,0 \pm$ & 19,0 & 1,2 & - & & - & - & - & $243,0 \pm 106,9$ & 2,7 & $128,0 \pm 22,0$ & 11,1 \\
\hline P. pillulare 5 & $558959,0 \pm 14$ & 491097,3 & 24,0 & $6658437,0 \pm 1$ & 198839,0 & 42,0 & $6658437,0 \pm$ & 1462126,0 & 20,0 & $360,0 \pm$ & 18,8 & 6,2 & - & - & - & - & - & - \\
\hline
\end{tabular}




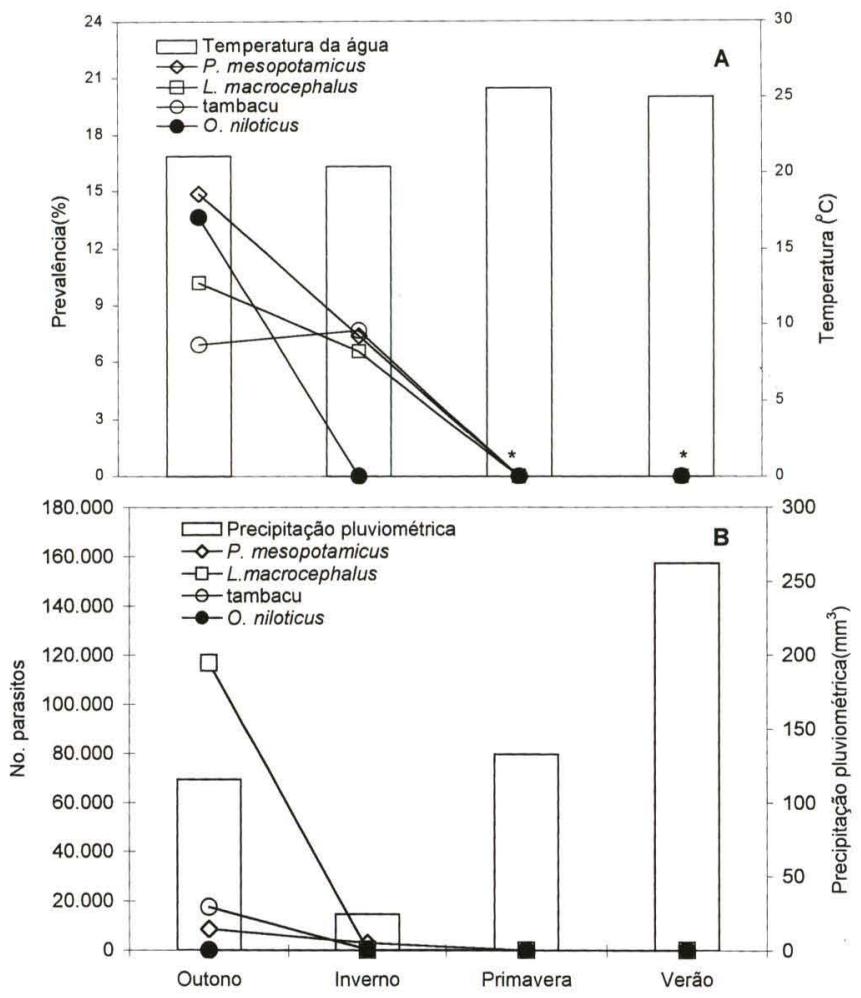

Fig. 2. Variação sazonal da temperatura da água, prevalência (A), precipitação pluviométrica e carga parasitária média do I. multifiliis $(B)$ em peixes examinados no período de abril de 1997 a março de 1999, em "pesque-pagues" do município de Franca, São Paulo. ( $\left.{ }^{*}\right)$ Diferença significativa $(P<0,05)$ na ocorrência entre as estações.

\section{Tratamentos e Mortalidade}

Durante o período de abril de 1997 a março de 1999, houve três casos de mortalidade após transporte de peixes para repovoamento de viveiros e outro envolvendo variações de temperatura. O primeiro caso ocorreu no mês de abril de 1997 (outono), com mortalidade de 150 espécimes de P. mesopotamicus infectados pelo protozoário $P$. pillulare e pelo monogenóide Anacanthorus penilabiatus Boeger, Husack \& Martins, 1995 (Dactylogyridae). Outro, foi em novembro de 1997 (primavera), com mortalidade de 20 L. macrocephalus infectados por monogenóide. A terceira ocorrência de mortalidade foi em abril de 1998, quando morreram $40 P$. mesopotamicus e 30 L. macrocephalus infectados por monogenea, Trichodina sp. e/ou I. multifiliis. O quarto caso, com mortalidade de 55 P. mesopotamicus foi observado no mês de outubro de 1998 (primavera), quando a temperatura chegou a $22^{\circ} \mathrm{C}$. Os peixes estavam parasitados por $P$. pillulare e $A$. penilabiatus.

No primeiro, segundo e quarto problemas de mortalidade os peixes sobreviventes receberam dois tratamentos com óxido de cálcio (Propeixe ${ }^{\mathbb{R}}$ da Campical), 

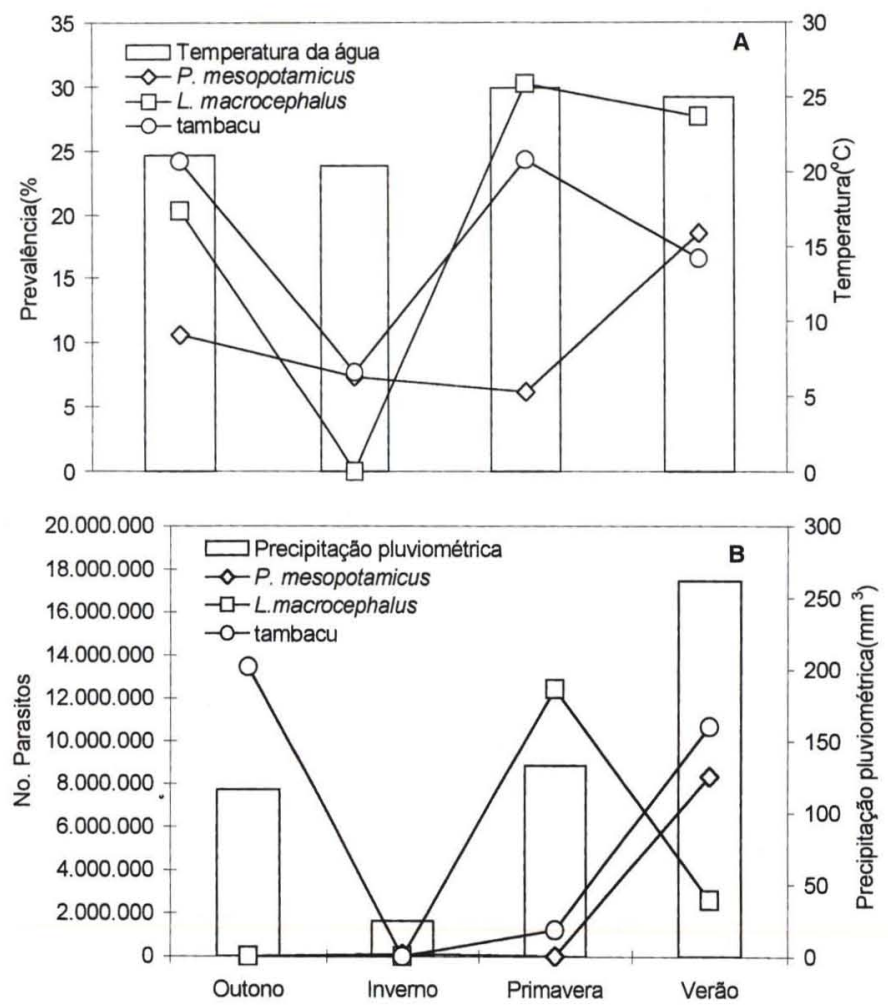

Fig. 3. Variação sazonal da temperatura da água, prevalência $(\mathbf{A})$, precipitação pluviométrica e carga parasitária média do $P$. pillulare $(B)$ em peixes examinados no período de abril de 1997 a março de 1999, em "pesque-pagues" do municipio de Franca, São Paulo. (*) Diferença significativa $(P<0,05)$ na ocorrência entre as estações.

em doses de $14 \mathrm{~g} / \mathrm{m}^{3}$, com intervalos de sete dias entre cada tratamento. No terceiro caso, os peixes foram submetidos a tratamento inicial com dose única de $14 \mathrm{~g} / \mathrm{m}^{3}$ de óxido de cálcio e, em seguida, a três tratamentos com $10 \mathrm{~mL}$ de formol $/ \mathrm{m}^{3}$ cada, com intervalos de dois dias entre cada tratamento.

Além desses problemas, em outra ocasião, após transporte de piauçús para repovoamento dos viveiros, vários peixes foram vistos agrupados na superfície e na entrada de água. O exame parasitológico revelou a presença de Monogenoidea e/ou P. pillulare nas brânquias dos peixes. Não houve, porém, ocorrência de mortalidade.

\section{DISCUSSÃO}

A análise dos parâmetros físico-químicos da água demonstrou similaridade entre os "pesque-pague" estudados, exceto no que se refere à condutividade elétrica dos viveiros, que apresentou maior oscilação no "pesque-pague" $2 \mathrm{RB}$. A qualidade da água, a temperatura, o pH e o oxigênio dissolvido estão diretamente correlacionados com a ocorrência de parasitos nos peixes (SINGHAL et al. 1986; RANZANI- 


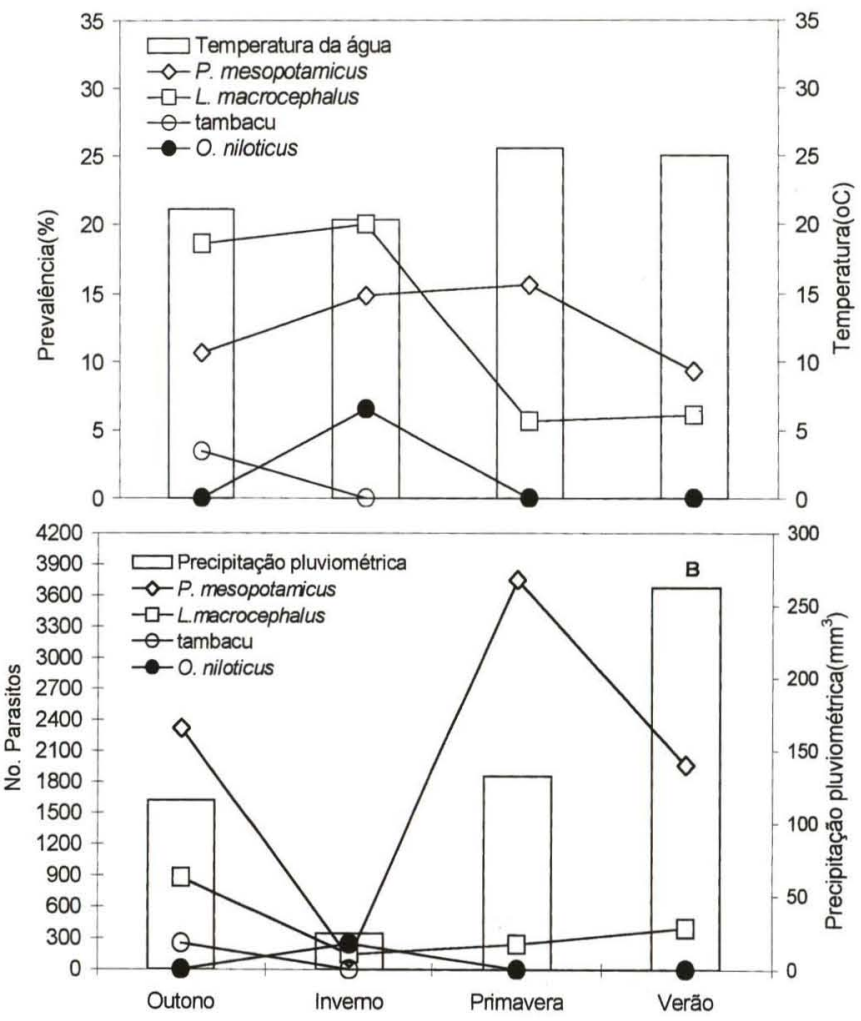

Fig. 4. Variação sazonal da temperatura da água, prevalência $(A)$, precipitação pluviométrica e carga parasitária média da Trichodina sp. (B) em peixes examinados no período de abril de 1997 a março de 1999, em "pesque-pagues" do município de Franca, São Paulo. ( ${ }^{*}$ ) diferença significativa $(P<0,05)$ na ocorrência entre as estações.

PAIVA 1997). Em ciprinídeos de cultivo intensivo, a associação de infecções severas por helmintos monogenóides, I. multifilis e Trichodina sp. (FIGUEIRA \& CECCARELLI 1991) ou por Trichodina sp., Argulus sp. e helmintos monogenóides (TOMEC et al. 1995), estão relacionadas à baixa qualidade da água e das condições sanitárias dos viveiros.

Neste estudo os protozoários encontrados foram o I. multifiliis, Trichodina sp., P. pillulare. Obervações em P. mesopotamicus e L. macrocephalus demonstram resultados similares (TAVARES-DiAs et al. 1999) enquanto, Trichodina sp. foi o protozoário comumente observado por BÉKESI (1992) em pisciculturas do nordeste brasileiro.

Nestas observações o percentual de infecção por I. multifiliis, em todas as espécies de peixes em "pesque-pague" avaliados, variou de 2,7\% a 11,1\%. A maior carga parasitária média foi observada em $L$. macrocephalus e a menor em $O$. niloticus. Similarmente EIRAS et al. (1995) demonstram baixa ocorrência de $I$. multifiliis em $P$. mesopotamicus, $O$. niloticus e $C$. carpio, cultivadas em "pesque- 
pague" do norte do Paraná, em condições sanitárias satisfatórias e densidade populacional relativamente baixa. Em P. mesopotamicus e Prochilodus lineatus Valenciennes, 1836 (Prochilodontidae) de piscicultura de Pindamonhangaba a prevalência do protozoário foi maior (EIRAS et al. 1995). CECCARELLI et al. (1990) descreveram infecção por I. multifiliis em C. macropomum, P. mesopotamicus, $C$. carpio, tambacu e carpa capim, com mortalidade de pacus e tambaquis.

O L. macrocephalus, P. mesopotamicus e C. carpio foram as espécies mais comprometidas pela infecção por Trichodina sp. quando comparados ao tambacu, $B$. cephalus, $T$. rendalli e a tilápia-do-Nilo, sendo que as maiores cargas parasitárias foram observadas em P. mesopotamicus. Nesta última espécie e em L. macrocephalus, a infecção por Trichodina sp. associada a monogenea e/ou I. multifilis provocou altas taxas de mortalidade. CECCARELLI et al. (1993) também relataram mortalidade em alevinos de P. mesopotamicus infectados por Trichodina sp. e por monogenea. Peixes portadores de infecções aguda ou subaguda tornam-se mais susceptíveis a outros agentes patógenos devido ao estresse. Estudos preliminares em P. mesopotamicus e L. macrocephalus de "pesque-pague", de Franca, demonstram que a prevalência desse protozoário é baixa (TAVARES-DiAs et al. 1999). Em peixes deste ensaio, as taxas de infecção por Trichodina sp. foram baixas se comparadas aos resultados descritos por EIRAS et al. (1995), em P. mesopotamicus e P. lineatus cultivados em pisciculturas de Pindamonhangaba e Pariquera-Açu, talvez devido ao pouco tempo de permanência dos peixes nos pesqueiros.

Neste ensaio as maiores prevalência e carga parasitária média por $P$. pillulare foram observadas em $L$. macrocephalus, $P$. mesopotamicus e tambacus de ambos os "pesque-pague". Porém, foi distinta a carga parasitária média desses hospedeiros nos dois "pesque-pague" estudados. Esse dinoflagelado ocorreu em baixo percentual e carga de infecção em $B$. cephalus, estando ausente nas tilápias e carpas. A ocorrência dessa parasitose estava acompanhada de alta densidade populacional nos viveiros, excesso de matéria orgânica e alterações na temperatura da água, sendo essa infecção associada a outras (MARTINS et al. no prelo) como observado também nessa oportunidade. Assim, nesta avaliação, tambacus, L. macrocephalus e $P$. mesopotamicus estavam infectados por $P$. pillulare e por helmintos monogenóides. Todavia, somente em $P$. mesopotamicus com elevada infecção por $P$. pillulare associada a infecção por $A$. penilabiatus ocorreu mortalidade.

De acordo com a literatura, a estrutura de comunidades em associações hospedeiro-parasito é determinada por fatores como a idade, a estrutura genética e o hábitat da população de hospedeiros, bem como por interações entre as espécies de parasitos (DOBSON \& KEYMER 1990 citado por ZUBEN 1997). A persistência dessas comunidades depende da relação entre a taxa de crescimento dos hospedeiros e a patogenicidade dos parasitos e para que essa persistência ocorra é necessário que a taxa de crescimento seja suficientemente alta para compensar a patogenicidade (DOBSON 1976 citado por ZUBEN 1997).

No Brasil, a ocorrência de $P$. pillulare foi observada somente a partir de 1996, em pisciculturas (MARTINs et al. no prelo) e em "pesque-pague" a partir de 1997 (TAVARES-DIAS et al. 1999), não havendo registro dessa parasitose em peixes de ambientes naturais. Portanto, os devidos cuidados devem ser tomados, no sentido 
de evitar a disseminação desse agente exótico para os rios brasileiros, uma vez que a piscicultura é o principal meio de dispersão de parasitos para ambientes naturais (ORSI \& Agostinho 1999). Devido a proximidade dos rios, os escapes de peixes para os cursos d'água podem ocorrer com os efluentes, o esvaziamento dos viveiros durante o manejo e, principalmente, pelo rompimento ou transbordamento em razão de cheias não previstas durante a construção (ORSI \& AGOSTINHO 1999).

Em L. macrocephalus, P. mesopotamicus e tambacu, na presente observação, as infecções por I. multifiliis ocorreram no outono e inverno, quando houve repovoamento dos viveiros. Mas em $O$. niloticus, somente nos meses de outono, demonstrando assim influência sazonal na ocorrência desse protozoário ciliado. Entretanto, em L. macrocephalus o maior número de parasitos ocorreu no outono. Tais resultados corroboram os dados da literatura, os quais sugerem que o $I$. multifiliis manifesta-se mais freqüentemente após o manejo dos peixes, em particular no período de baixas temperaturas (CECCARELLI et al. 1990; THATCHER \& Brites-Neto 1994; Martins \& Romero 1996). Em truticulturas da Dinamarca, a maior ocorrência de I. multifiliis ocorreu nos meses de verão e outono (BUCHMANN et al. 1995; BUCHMANN \& BRESCIANI 1997), quando a temperatura máxima chegou a $16-20^{\circ} \mathrm{C}$ (BUCHMANN \& BRESCIANI 1997), pois as condições ótimas de temperatura para o parasito completar seu ciclo, em hábitat de clima temperado, varia de 20 a $25^{\circ} \mathrm{C}$ (MEYER 1970; FrANCIS-FLOYD \& NoGA 1994; KLESIUS \& ROGERS 1995). Em contrapartida, em piscicultura da India a ocorrência não é influenciada pela sazonalidade, pois as infecções ocorrem durante todo ano (SINGHAL et al. 1986). BAUER \& KARIMOV (1990), sugerem que em hábitat onde a temperatura da água é constante, como nas regiões tropicais, as variações das populações de parasitos não são significativas e, portanto não sofrem influência da temperatura da água.

No presente trabalho, a prevalência de Trichodina sp. não apresentou variação sazonal significativa. Em tambacu esses protozoários foram observados somente nos meses de outono, e em O. niloticus, no inverno. Em L. macrocephalus e em P. mesopotamicus o número de Trichodina sp. declinou no inverno e, no $P$. mesopotamicus voltou a crescer na primavera. De acordo com a literatura a infecção por Trichodina sp. ocorre geralmente no inverno (MEYER 1970). No Uruguai, a maior ocorrência dessa parasitose em Mugil liza Valenciennes, 1836 (Mugillidae) foi observada no inverno (CARNEVIA et al. 1988) e em carpa prateada $H$. molitrix da Ïndia, no inverno e primavera (SingHAL et al. 1986) mas, percentuais elevados de prevalência também foram observados nas demais estações. Por outro lado, em Gasterosteus aculeatus Linnaeus, 1776 (Gasterosteidae) cultivados na Escócia (Chappell 1969) e em Mugil platanus Günther, 1880 (Mugillidae) de região estuariana brasileira (RANZANI-PAIVA et al. 1997) a infeção não demonstrou influência sazonal.

Nos "pesque-pague" de Franca a prevalência de $P$. pillulare não foi significativamente influenciada pela sazonalidade. Porém, o número de parasitos, em tambacu foi menor no inverno e, em $L$. macrocephalus no outono e inverno, aumentando na primavera, quando a temperatura aumentou. Em $P$. mesopotamicus as maiores cargas parasitárias ocorreram durante o verão sendo menores no outono. MARTins et al. (no prelo) verificaram que $73,3 \%$ dos casos de infecção por $P$. 
pillulare ocorrem no período de outono e inverno, com temperaturas entre $17 \mathrm{e} 24^{\circ} \mathrm{C}$, pois o ciclo de vida desse parasito pode ser completado em 10 a 14 dias, em temperatura de $23 \mathrm{a} 25^{\circ} \mathrm{C}$. A elevada freqüência de parasitos em novos hospedeiros, indicam que esses são susceptíveis ao novo parasito e que há ausência de equilíbrio nos novos sistemas parasito-hospedeiro (POJMANSKA \& CHABROS 1993).

Os resultados deste estudo demonstram que apesar da ocorrência de epizootias com altas taxas de mortalidade no início e durante os meses mais frios do ano, não há aumento da tensão ambiental de infestação por parasitos, uma vez que nessa época a carga prasitária dos hospedeiros é a mais baixa do ano. Tais surtos seriam devidos às más condições da água e ao manejo inadequado como indutores de estresse, que atuariam como elementos predisponentes às enfermidades. A queda da temperatura ambiente, com conseqüente redução da taxa metabóllica desencadearia as enfermidades, mais pela redução da resistência do hospedeiro do que pela alta prevalência de parasitos. Estes, sejam de peixes, como de mamíferos de interesse zootécnico, experimentam aumento do período do seu ciclo biológico nos meses mais frios do ano, com tendência de redução da tensão ambiental de infestação.

\section{CONCLUSÕES}

Os resultados indicam que nas brâquias dos peixes necropsiados houve prevalência de Ichthyophthirius multifiliis, Trichodina sp. e Piscinoodinium pillulare. Por ordem decrescente, o grau de suscetibilidade dos hospedeiros aos parasitos foi $L$. macrocephalus, $P$. mesopotamicus, tambacu, $C$. carpio, $O$. niloticus, $T$. rendalli e $B$. cephalus. Em todas as espécies necropsiadas, a maior prevalência de I. multifiliis foi no período de outono e/ou inverno. Não houve interferência sazonal estatisticamente significativa em nenhuma das espécies de peixe para Trichodina sp. e $P$. pillulare.

\section{REFERÊNCIAS BIBLIOGRÁFICAS}

Bauer, O.N. \& S.B. Karimov. 1990. Patterns of parasitic infections of fishes in a water body with constant temperature. Jour. Fish Biol 36: 1-8.

BĖKÉSI, L. 1992. Evaluation of data on ichthyopathological analyses in the Brazilian Northeast. Ciên. Cult. 44 (6): 400-403.

BuchmanN, K.; A. Uldal \& H.C.K. Lyholt. 1995. Parasite infections in danish trout farms. Acta Vet. Scand. 36: 283-298.

BuchmanN, K. \& J. Bresciani. 1997. Parasitic infections in pond-reared rainbow trout Oncorhynchus mykiss in Denmark. Dis. Aquat. Organ. 25: 125-138.

Bush, A.O.; K.D. LAFFERTY; J.M. LotZ; \& A. W.ShostAK. 1997. Parasitology meets ecology on its own terms: Margolis et al. Revisited. Jour. Parasitol. 83 (4): 575-583.

CARnevia, D.; R. Mazzoni; O. Areosa; T. EAstMan \& D. Lorenzo. 1988. Variaciones estacionales de diversas parasitosis em la lisa Mugil liza - Val. 1836 del Rio de La Plata. An. VI Simp. Latinoamer. e V Simp. Brasil. Aqüicul., Florianópolis, p. 299-304.

Ceccarelli, P.S.; L.B. Figueir^; C.L.B. Ferraz Lim^ \& C.A. OliveirA. 1990. Observações sobre a ocorrência de parasitos no CEPTA entre 1983 e 1990. Bol. Téc. CEPTA 3: 43-55.

Ceccarelli P.S.; R.C.G. Alcântara-Rocha \& J.S.C. Melo. 1993. Efeito do formaldeído sobre a Trichodina sp. e Linguadactyloides sp. em alevinos de pacu, Piaractus mesopotamicus Holberg, 1887. Bol. Téc. CEPTA 6 (2): 23-30.

CHAPPELL, L.H. 1969. The parasites of the three-spined stickleback Gasterosteus aculeatus L. from a Yorkshire pond. II. Variation of parasite fauna with sex and size of fish. Jour. Fish Biol 1: 339-347. 
Eiras, J.C.; M.J.T. Ranzani-Paiva; C.M. Ishikawa; A.C. Alexandrino \& A.C. Eiras. 1995. Ectoparasites of semi-intensively farmed tropical freshwater fish Piaractus mesopotamicus, Prochilodus lineatus and Colossoma macropomum in Brazil. Bull. Eur. Ass. Fish Pathol 15 (5): 148-151.

Eiras, J.C.; G.C. P^vanelli; J.A. \& R.M. TAKemoto. 2000. Métodos de estudo e técnicas laboratoriais em parasitologia de peixes. Maringá, Eduem, 171p.

FIGUEIRA, L.B. \& P.S. CECCARELLI. 1991. Observações sobre a presença de ectoparasitas em pisciculturas tropicais de interior (CEPTA e região). Bol. Téc. CEPTA 4 (1): 57-65.

Francis-Floyd, R. \& E.J. NogA. 1994. Medical management of channel catfis. Part I. Types of skin and gill pathogens. Compend. Contin. Educ. Pract. Vet. 16 (6): 808-814.

KLesius, P. \& W. Rogers. 1995. Parasitism of catfish and other farm-raised food fish. J.A.V.M.A. 207 (11): 1473-1478.

Martins, M.L. 1998. Doenças infecciosas e parasitárias de peixes. Jaboticabal, FUNEP, $2^{a}$ ed., 65p.

Martins, M.L. \& N.G. Romero. 1996. Efectos del parasitismo sobre el tejido branquial en peces cultivados: estudio parasitologico e histopatologico. Revta bras. Zool. 13 (2): 489-500.

Martins, M.L.; J.R.E. Moraes; P.M. Andrade; S.H.C. Schalch \& F.R. Moraes. (in press). Piscinoodinium pillulare (Schäperclaus 1954) Lom, 1981 (Dinoflagellida) infection in cultivated freshwater fish from northeast region of São Paulo State, Brazil. Parasitological and pathological aspects. Rev. Brasil. Biol. 61 (3).

MEYER, F.P. 1970. Seasonal fluctuations in the incidence of disease on fish farms. Symp. Amer. Fish. Soc. Dis. Shell Fish Special, Washington, 5: 21-29.

ORSI, M.L. \& A.A. AgOSTINHO. 1999. Introdução de espécies de peixes por escapes acidentais de tanques de cultivo em rios da Bacia do Rio Paraná, Brasil. Revta bras. Zool. 16 (2): 557-560.

Pojmanska, T. \& M. Chabros. 1993. Parasites of common carp and three introduced cyprinid fish in pond culture. Acta Parasitol. 38 (3):101-108.

Ranzani-Paiva, M.J.T. 1997. Fungos parasitos de peixes. Biológico, São Paulo, 59 (1): 57-59.

Ranzani-Paiva, M.J.; C.M. Ishikawa; B.E.S. Campos \& A.C. Eiras. 1997. Haematological characteristics associated with parasitism in mullets, Mugil platanus Günther, from the estuarine region of Cananéia, São Paulo, Brazil. Revta bras. Zool. 14 (2): 329-339.

Singhal, R.N.; S. JeET \& R.W. Davies. 1986. The relationships between changes in selected physico-chemical properties of water and the occurrence of fish parasites in Haryana, India. Trop. Ecol. 27: 1-9.

SteEl, R.G.D. \& J.H. TorRIE. 1980. Principles and procedures of statistics. New York, McGraw Hill, $2^{\text {nd }}$ ed., 633p.

Tavares-Dias, M.; S.H.C. Schalcii; M.L. Martins; E.D. Silva; F.R. Moraes \& D. Perecin. 1999. Hematologia de teleósteos brasileiros com infecção parasitária. I. Variáveis do Leporinus macrocephalus Garavello \& Britski, 1988 (Anostomidae) e Piaractus mesopotamicus Holmberg, 1887 (Characidae). Acta Scientiarum 21: 337-342.

Tavares-Dias, M.; C.A.P. Rodrigues; F.R. Moraes; M.L. Martins \& A.E. Santana (in press). Haematological changes in Oreochromis niloticus Linnaeus, 1758 (Osteichthyes: Cichlidae) with gill ichthyophthiriasis and saprolegniosis. Bol. Inst. Pesca, São Paulo..

THATCHER, V.E. 1991. Amazon fish parasites. Amazoniana 11 (3/4): 263-572.

Th^tcher, V.E. \& J. Brites-Neto.1994. Diagnósitco, prevenção e tratamento das enfermidades de peixes neotropicais de água doce. Rev. Brasil. Med. Vet. 16 (3): 111-128.

Tomec, M.; M. Hacmanjek; Z. Teskeredzic; E. Teskeredzic \& R. Coz-Rakovac. 1995. Kvaliteta vode i ektoparazitarne bolesti ciprinidnih riba. Ribarstvo 53 (4): 129-139.

ZUBEN, C.J. 1997. Implicações da agregação espacial de parasitas para dinâmica populacional na interação hospedeiro-parasita. Rev. Saúde Públ. 31 (5): 523-530. 